\title{
Effects of a single bout of power exercise training on ambulatory blood pressure in older adults with hypertension: A randomized controlled crossover study
}

\author{
Renato P Schimitt ${ }^{\mathrm{a}, \mathrm{b}}$, Leandro O Carpes ${ }^{\mathrm{a}, \mathrm{b}}$, Lucas B Domingues ${ }^{\mathrm{a}, \mathrm{b}}$, Hirofumi Tanaka ${ }^{\mathrm{c}}$, \\ Sandra C Fuchs ${ }^{a}$, Rodrigo Ferrari ${ }^{a, b, *}$ \\ ${ }^{a}$ Postgraduate Program in Cardiology, Universidade Federal do Rio Grande do Sul, Porto Alegre, RS, Brazil \\ ${ }^{\mathrm{b}}$ Sports and Exercise Training Study Group, Hospital de Clínicas de Porto Alegre, RS, Brazil \\ ${ }^{\mathrm{c}}$ Cardiovascular Aging Research Laboratory, The University of Texas at Austin, Austin, TX, USA
}

\section{A R T I C L E I N F O}

\section{Keywords:}

Post-exercise hypotension

Strength training

Aging

Resistance exercise

\begin{abstract}
A B S T R A C T
Objective: To evaluate the effect of a single bout of power exercise training (PT) on office and ambulatory blood pressure (BP).

Methods: Twenty-four older adults with essential hypertension participated in two experimental sessions in a randomized order: the PT composed of 3 sets of 8-10 repetitions in 5 power training exercises and the nonexercise control at seated rest (Con). Both experimental sessions lasted $40 \mathrm{~min}$. Office BP was measured continuously for $1 \mathrm{~h}$ in the laboratory and $24 \mathrm{~h}$ BP through ambulatory blood pressure monitoring.

Results: Compared with Con, office systolic/diastolic BP decreased after PT (Systolic BP: $10 \mathrm{mmHg}, \mathrm{p}<0.001$; Diastolic BP: $4 \mathrm{mmHg}, \mathrm{p}=0.015)$. A trend toward decrease $(\mathrm{p}=0.06)$ was found in diastolic ambulatory BP during daytime $(2 \mathrm{mmHg} ; \mathrm{p}=0.062)$ and nighttime $(3 \mathrm{mmHg} ; \mathrm{p}=0.063)$ after PT. No differences were found between PT and Con sessions for systolic and mean ambulatory BP.

Conclusion: A single bout of PT decreases office BP but this hypotensive effect is not sustained under ambulatory conditions in older patients with essential hypertension.
\end{abstract}

\section{Introduction}

Skeletal muscle mass, muscle strength and power, and physical independence decrease with advancing age. ${ }^{1,2}$ These declines are associated with development of cardiovascular diseases and mortality., Hypertension is one of the most important modifiable risk factors for developing cardiovascular disease and increases its prevalence and severity throughout lifespan. ${ }^{5-7}$ Regular physical exercise is a cornerstone intervention to attenuate and prevent the age-related increases in hypertension $^{8}$ and increase functional independence. ${ }^{9}$ In particular, resistance training has emerged as one of the best strategies to improve neuromuscular function and the capacity to perform daily living activities in the older populations. ${ }^{10}$ Moreover, results of a meta-analysis suggest resistance training as viable stand-alone antihypertensive lifestyle therapy. ${ }^{11}$

After a single bout of exercise, BP decreases when compared with the baseline values of the same session or if compared to a usual day without exercise, a phenomenon termed post-exercise hypotension (PEH). ${ }^{12}$ This acute post-exercise reduction of BP may predict the extent of BP lowering after chronic training interventions, since important correlations between the acute and chronic BP reduction after exercise were found. ${ }^{15,16}$ To improve its clinical relevance, PEH should be followed and assessed throughout long periods ( $24 \mathrm{~h}$ ) and under usual or ambulatory conditions. ${ }^{17}$ However, few studies have assessed the effects of resistance exercise on ambulatory $\mathrm{BP}$ and none of them used older participants with hypertension. ${ }^{18}$ It remains uncertain how long a single session of resistance exercise exerts PEH effects among older individuals with hypertension under ambulatory conditions including daily activities and sleeping.

The available literature suggests that exercise prescription should include power exercises based on greater enhancements in functional outcomes after power exercise training when compared with traditional resistance training in older adults. ${ }^{9,19}$ Power exercise training is performed at lower intensities $(30-50 \%$ of one repetition maximum or

\footnotetext{
* Corresponding author at: Hospital de Clínicas de Porto Alegre, Rua Ramiro Barcelos 2350, 90035-903, Porto Alegre, RS, Brazil.

E-mail addresses: rod.ferrari84@gmail.com, rodrifsilva@hcpa.edu.br (R. Ferrari).
} 
1-RM), lower volumes per set (up to ten repetitions) and are not set to failure or volitional exhaustion. These exercise characteristics are attractive to higher risk populations such as older patients with hypertension who demonstrate compromised cardiovascular and musculoskeletal functions. The number of repetitions per set greatly and adversely affects BP response during exercise when resistance training is performed to volitional exhaustion. ${ }^{20-22}$

To the best of our knowledge, studies investigating an effect of a single bout of power exercise training (PT) on BP are scarce, and the available studies only assessed BP under office conditions, ${ }^{23,24}$ and no data are available on the effects of power exercise training on ambulatory BP. Accordingly, the aim of the present study was to evaluate the effect of PT on office and $24 \mathrm{~h}$ blood pressure in older adults with hypertension. The working hypothesis was that a single bout of power exercise would decrease both office and $24 \mathrm{~h}$ BP in comparison to a non-exercising control session.

\section{Materials and methods}

\subsection{Study design and participants}

A randomized controlled clinical trial with crossover design was performed in order to evaluate the effect of PT on office and $24 \mathrm{~h} \mathrm{BP}$ of hypertensive patients. The protocol followed the CONSORT guidelines for non-pharmacological treatment. ${ }^{25}$ Participants performed two experimental sessions in a random order: a power exercise training session (PT) and a non-exercising control session (Con). The randomization list composed of random block sizes of four participants was generated by an epidemiologist using a computer software. This epidemiologist did not participate in the recruitment or assignment to the experimental sessions. Participants and the research team were blinded to the randomization list until the moment of assignment.

Men and women aged 60-75 years with previously diagnosed hypertension by a physician were recruited if they had not engaged in structured exercise programs in the last 3 months prior to the start of the trial. Exclusion criteria included previous diagnosis of ischemic heart disease, heart failure, current smokers or ex-smokers for less than 6 months, body mass index over $39.9 \mathrm{~kg} / \mathrm{m}^{2}$, musculoskeletal problems that prohibit them from exercising, and diabetes with retinopathy.

The study was conducted from 12 June 2018 to 20 July 2019 at a tertiary referral hospital in southern Brazil. All participants read and signed an informed consent form before beginning the study. Participation was voluntary and all ethical principles of confidentiality and data protection were followed. The study protocol was conducted according to the principles of the Declaration of Helsinki and in compliance with the Brazilian legal and regulatory framework for research involving human beings (NR 466/12). The study protocol was approved by the Institutional Review Board of Hospital de Clínicas de Porto Alegre, Brazil, and registered on clinicaltrials.gov under identifier number NCT03615625.

\subsection{Preliminary sessions}

Each participant completed a clinical screening, resting electrocardiogram, manual BP and anthropometric measurements in the research laboratory. Participants were then instructed to attend three preliminary sessions on 3 different days separated by 48-96 hours. On the first and second sessions, the research team performed office BP assessments in duplicate using an automated BP device (Dinamap, Critikon, EUA). The stability of BP was confirmed in this run-in period. The participants were also familiarized with power exercises involved in PT and maximal strength testing during the first two sessions. Since most participants included in the study had no previous experience with power exercise training, we implemented two familiarization sessions in order to ensure that the participants' properly perform the prescribed exercises. During the third preliminary session, participant's maximal strength was assessed using one repetition maximal strength (1-RM) test. Before the test, resting BP was assessed after 20 min of rest in supine position and these values were used to describe the baseline characteristics of participants.

Height and body mass were assessed by a stadiometer and a balance scale (FILIZOLA, Brasil) and used to calculate body mass index (BMI). Waist circumference was assessed using an inelastic standardized measure-tape (Cescorf, Brazil) at the middle point between upper iliac crest and the lower costal rib in the horizontal plane. To determine the proper cuff size used in the BP monitoring, arm circumference was assessed at middle point between acromion and the cubital fossa on the non-dominant arm.

Maximal dynamic strength was evaluated using the one repetition maximum test (1-RM) in 5 resistance exercises: leg press, bench press, knee extension, upright row, and knee flexion. A specific warm-up composed of 2 sets of 10 and 5 repetitions, using $50 \%$ and $75 \%$ of estimated 1-RM load was performed prior to the test. After the first attempt, the load was adjusted through Lombardi coefficients, if necessary. Each participant's 1RM was determined with no more than three attempts with a five-minute recovery between attempts and a twominute recovery between exercises. These results were used to determine the intensity or load of the experimental sessions. The tests were conducted by the same trained investigator with previous experience in this method.

\subsection{Experimental sessions}

Participants performed two experimental sessions: power exercise training (PT) and non-exercising control at seated rest (Con). Participants were instructed to avoid physical exercise for $24 \mathrm{~h}$ before the experimental sessions, keep the usual dietary intake, avoid alcohol, coffee, and other stimulant substances before the experimental sessions and have the same meal $4 \mathrm{~h}$ before each session. Participants maintained their current antihypertensive medications throughout the trial.

Both experimental sessions started between 2-3 PM (at the same time of day to account for potential diurnal variation in BP and residual effects of antihypertensive medications) and lasted approximately $2 \mathrm{~h}$. Each session was composed of $20 \mathrm{~min}$ of rest in the supine position, $40 \mathrm{~min}$ of PT or Con protocols, and $60 \mathrm{~min}$ of rest in supine position after protocols. Standardized office BP and heart rate assessments were performed before, immediately after each exercise during PT session or in intervals of $8 \mathrm{~min}$ during Con, and during the first hour (in intervals of $15 \mathrm{~min}$ ) after the experimental sessions using an automatic oscillometric device (Dinamap 1846 SX/P; Critikon, FL, USA). Afterwards, participants underwent 24 - $\mathrm{h}$ ambulatory blood pressure monitoring (90702, Spacelabs Medical, EUA).

The PT was composed of 3 sets of $8-10$ repetitions of 5 exercises performed in the following order: leg press, bench press, knee extension, upright row, and knee flexion, using an intensity corresponding to $50 \%$ of 1-RM and two-minute intervals between sets and exercises. The concentric phase of exercises during each repetition was performed "as fast as possible" while the eccentric phase lasted 1-2 seconds. During the Con, the participants remained seated rest on the same exercise machines, but without any exercise.

Office BP was assessed under laboratory conditions according to the VII Brazilian National Guidelines in Cardiology. ${ }^{26}$ Participants were instructed to remain in silence without using any electronic device (i.e., smartphones, notebooks). A proper-sized cuff was placed in the arm about $2 \mathrm{~cm}$ from the antecubital fossa. Measurements of BP were performed on both arms with a time difference of $1 \mathrm{~min}$ between procedures. Measurements were performed twice in the arm with the highest $\mathrm{BP}$ values. The mean of these BP values was used to represent office BP. The arm with the highest BP values was used for the subsequent assessments.

The primary outcome of the present study was the difference in $24 \mathrm{~h}$ ambulatory BP assessed after each experiment session. Measurements of 
BP were taken every $15 \mathrm{~min}$ at daytime and every $20 \mathrm{~min}$ at nighttime. The first daytime period started between 4-5 PM (immediately after laboratory session), nighttime between $11 \mathrm{PM}$ and $7 \mathrm{AM}$, and the second daytime finished at $5 \mathrm{PM}$ on the day after the experimental session. Participants filled a diary about activities, symptoms, sleep and wake-up time. Each exam was considered valid when at least $70 \%$ of the expected readings were available and recorded. ${ }^{27}$

\subsection{Statistical analyses}

Sample size was estimated according to the results of a previous study using a similar study design. ${ }^{28}$ An initial sample size of 24 individuals with hypertension, allowing a dropout rate of $10 \%$, would be able to detect a difference of $5 \mathrm{mmHg}$ in systolic BP between the protocols with $80 \%$ of statistical power and a type I error rate of $5 \%$. WinPepi software calculator was used to estimate the sample size.

Data were entered in duplicate by three different researchers and expressed as means and standard deviation for variables with normal distribution or medians and interquartile range for non-normal distributions and $95 \%$ confidence intervals (CI95 \%). Generalized Estimating Equations (GEE) analysis was used to compare main effects between experimental sessions using absolute BP values, assessing the condition ( 2 session: PT and Con) by time (3 factors: day-time 1, night-time, and day-time 2). Post-hoc comparisons were performed with Bonferroni test. Statistical significance was set at $\mathrm{P}<0.05$, and a borderline significance was detected for P-values ranging from 0.05 to 0.10 . All statistical analyses were performed using SPSS Statistics for Windows, version 22.0 (IBM, Armonk, NY).

\section{Results}

A flowchart of the experiments is presented in Fig. 1. Participants' characteristics at baseline assessed during the preliminary sessions are shown in Table 1. Overall, participants were overweight and had been
Table 1

Selected participant characteristics.

\begin{tabular}{ll}
\hline Age, years (SD) & $66.7(4.3)$ \\
Sex, n (\%) & \\
Men & $12(50)$ \\
Women & $12(50)$ \\
Race/Etnicity, n (\%) & \\
White & $20(83.3)$ \\
Black & $3(12.5)$ \\
Asian & $1(4.2)$ \\
Anti-hypertensive medications, median (range) & $2(1-4)$ \\
Diuretics, n (\%) & $19(79.2)$ \\
$\beta$ blockers, n (\%) & $17(70.8)$ \\
Angiotensin converting enzyme inibitors, n (\%) & $8(33.3)$ \\
Angiotensin receptor antagonists, n (\%) & $14(58.3)$ \\
Calcium channel blockers, n (\%) & $19(79.2)$ \\
$\alpha-2$ agonists, n (\%) & $1(4.2)$ \\
Combined Therapy, n (\%) & $23(95.8)$ \\
Anthropometric measures & \\
Body weight, kg (range) & $76(69-87)$ \\
Height, cm (range) & $162(156-169)$ \\
BMI, kg/m ${ }^{2}$ (SD) & $29.7(3.7)$ \\
Waist circunference, cm (range) & $96.5(87.5-106.8)$ \\
Waist-height ratio (range) & $0.6(0.55-0.66)$ \\
Hemodynamic measures & \\
Systolic BP, mmHg (SD) & $132(12)$ \\
Diastolic BP, mmHg (SD) & $76(8)$ \\
Mean BP, mmHg (SD) & $96(8)$ \\
Heart rate, bpm (SD) & $66(11)$ \\
RPP, mmHg/bpm (SD) & $8824(1728)$ \\
Muscle strength tests & \\
1-RM Leg Press, kg (SD) & $137.6(57.0)$ \\
1-RM Knee Extension, kg (SD) & $86.5(28.2)$ \\
1-RM Knee Flexion, kg (SD) & $53.5(18.6)$ \\
1-RM Bench Press, kg (SD) & $37.9(14.9)$ \\
1-RM Upright row, kg (SD) & $29.8(11.6)$ \\
\hline
\end{tabular}

Means \pm SD for parametric data and medians \pm interquartile interval for nonparametric data; $\mathrm{BMI}=$ body mass index; $\mathrm{BP}$ : blood pressure; $\mathrm{RPP}=$ rate-pressure product; $1-\mathrm{RM}=$ one repetition maximal strength.

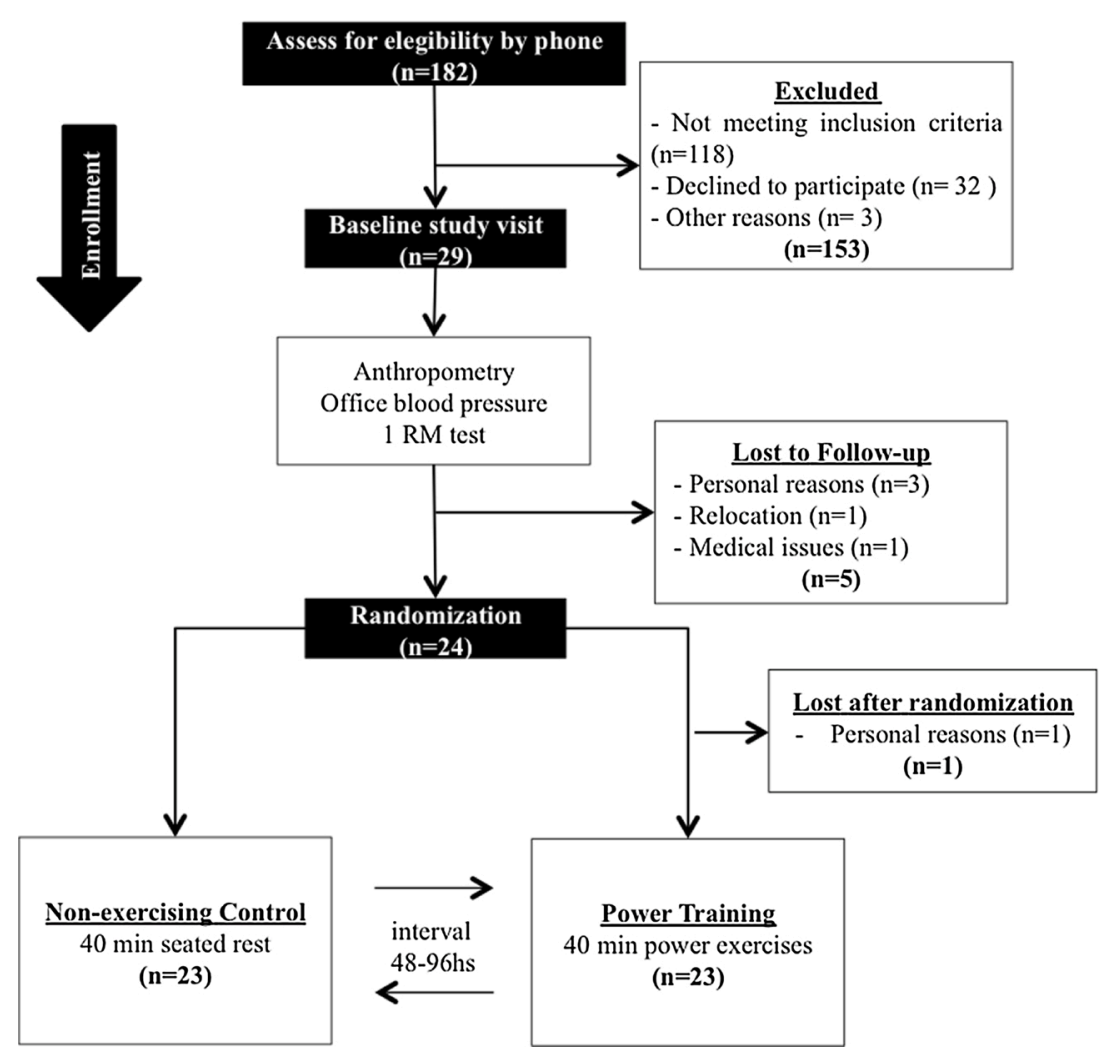

Fig. 1. Participant flow chart of the present study. 1-RM = one-repetition maximal strength. 
taking two anti-hypertensive drugs. There were no reported adverse events during the PT session. The quality of the ambulatory BP recorded was considered satisfactory in all patients. Analyses on the activity diary filled out by the participants showed a similar pattern in both experimental sessions days, with similar domestic activities performed, a similar pattern in meal times and medications. Additionally, no adverse symptoms or difficulties to sleep were reported by the participants.

Time vs. session interaction was found for systolic ( $p<0.001)$, mean ( $p<0.001$ ), and diastolic ( $p<0.001)$ office BP. Baseline BP values were similar for systolic BP (132 vs. $131 \mathrm{mmHg} ; \mathrm{p}=0.616)$, mean BP (91 vs. $95 \mathrm{mmHg} ; \mathrm{p}=0.509$ ), and diastolic $\mathrm{BP}$ (76 vs. $75 \mathrm{mmHg}$; $\mathrm{p}=0.576$ ) between PT and Con. During the first hour in the research laboratory, office systolic $(-10 \mathrm{mmHg}$, CI95 \%: -15 to $-5 ; \mathrm{p}<0.001)$, mean ( $-6 \mathrm{mmHg}$, CI95 \%: -9 to $-3 ; \mathrm{p}=0.001)$, and diastolic BP $(-4 \mathrm{mmHg}$, CI95 \%: -7 to $-1 ; \mathrm{p}=0.015)$ decreased significantly after PT when compared with Con.

Ambulatory BP data are presented in Table 2. No differences were found between PT and Con sessions for day-time, night-time and total $24 \mathrm{~h}$ systolic and mean BP. A borderline significance reduction in the first day-time $(\mathrm{p}=0.063)$ and night-time $(\mathrm{p}=0.062)$ diastolic BP was found after the PT session when compared with Con. There were no differences in $24 \mathrm{~h}$ diastolic BP between PT and Con sessions.

Table 3 describes the hemodynamic response data during the experimental protocols. A significant increase in mean BP was seen after upright row exercise when compared to the corresponding baseline value. Rate-pressure product was consistently elevated during PT when compared with Con.

\section{Discussion}

Office BP has often been used to diagnose hypertension. However, due to a number of methodological issues including white coat hypertension, ambulatory BP has become the gold-standard measurement for

Table 2

Ambulatory $(24 \mathrm{~h})$ blood pressure in the power exercise training and nonexercising control sessions.

\begin{tabular}{|c|c|c|c|c|}
\hline & Control & Power Training & Delta & $\begin{array}{l}\text { p-value } \\
\text { PT vs. } \\
\text { Con }\end{array}$ \\
\hline \multicolumn{5}{|l|}{ Systolic BP } \\
\hline $24-\mathrm{h}$ & $\begin{array}{l}131.5 \pm 2.7 \\
(126-137)\end{array}$ & $\begin{array}{l}131.4 \pm 2.3 \\
(127-136)\end{array}$ & $\begin{array}{l}0.1 \pm 1.6(-2.9 \\
\text { to } 3.1)\end{array}$ & 0.931 \\
\hline Day-time 1 & $\begin{array}{l}138.9 \pm 2.7 \\
(134-144)\end{array}$ & $\begin{array}{l}137.4 \pm 3.0 \\
(132-144)\end{array}$ & $\begin{array}{l}1.4 \pm 1.9 \\
(-5.2-2.3)\end{array}$ & 0.448 \\
\hline Night-time & $\begin{array}{l}126.4 \pm 3.2 \\
(120-133)\end{array}$ & $\begin{array}{l}123.8 \pm 2.9 \\
(118-130)\end{array}$ & $\begin{array}{l}2.6 \pm 2.1(-6.9 \\
\text { to } 1.6)\end{array}$ & 0.375 \\
\hline Day-time 2 & $\begin{array}{l}131.4 \pm 2.8 \\
(126-137)\end{array}$ & $\begin{array}{l}131.0 \pm 2.5 \\
(126-136)\end{array}$ & $\begin{array}{l}0.3 \pm 1.9(-3.4 \\
\text { to } 4.0)\end{array}$ & 0.870 \\
\hline \multicolumn{5}{|l|}{ Mean BP } \\
\hline $24-\mathrm{h}$ & $\begin{array}{l}96.0 \pm 2.0 \\
(92-100)\end{array}$ & $\begin{array}{l}95.1 \pm 1.8 \\
(91-99)\end{array}$ & $\begin{array}{l}0.9 \pm 1.2(-3.3 \\
\text { to } 1.4)\end{array}$ & 0.432 \\
\hline Day-time 1 & $\begin{array}{l}102.2 \pm 2.1 \\
(98-106)\end{array}$ & $\begin{array}{l}100.3 \pm 2.2 \\
(96-105)\end{array}$ & $\begin{array}{l}1.8 \pm 1.3(-4.4 \\
\text { to } 0.6)\end{array}$ & 0.149 \\
\hline Night-time & $\begin{array}{l}91.1 \pm 2.3 \\
(87-96)\end{array}$ & $\begin{array}{l}88.9 \pm 1.9 \\
(85-93)\end{array}$ & $\begin{array}{l}2.2 \pm 1.7(-5.6 \\
\text { to } 1.1)\end{array}$ & 0.201 \\
\hline Day-time 2 & $\begin{array}{l}96.3 \pm 2.2 \\
(92-101)\end{array}$ & $\begin{array}{l}95.5 \pm 1.9 \\
(92-99)\end{array}$ & $\begin{array}{l}0.7 \pm 1.4(-3.4 \\
\text { to } 2.0)\end{array}$ & 0.599 \\
\hline \multicolumn{5}{|l|}{$\begin{array}{l}\text { Diastolic } \\
\text { BP }\end{array}$} \\
\hline $24-\mathrm{h}$ & $\begin{array}{l}77.2 \pm 1.9 \\
(73-81)\end{array}$ & $\begin{array}{l}76.1 \pm 1.9 \\
(72-80)\end{array}$ & $\begin{array}{l}1.0 \pm 0.9(-2.9 \\
\text { to } 0.8)\end{array}$ & 0.279 \\
\hline Day-time 1 & $\begin{array}{l}82.7 \pm 2.1 \\
(79-87)\end{array}$ & $\begin{array}{l}80.6 \pm 2.3 \\
(76-85)\end{array}$ & $\begin{array}{l}2.0 \pm 1.1(-4.2 \\
\text { to } 0.1)\end{array}$ & 0.063 \\
\hline Night-time & $\begin{array}{l}73.2 \pm 1.9 \\
(69-77)\end{array}$ & $\begin{array}{l}69.7 \pm 2.1 \\
(66-74)\end{array}$ & $\begin{array}{l}3.4 \pm 1.8(-7.1 \\
\text { to } 0.1)\end{array}$ & 0.062 \\
\hline Day-time 2 & $\begin{array}{l}79.0 \pm 2.1 \\
(75-83)\end{array}$ & $\begin{array}{l}77.3 \pm 2.0 \\
(73-81)\end{array}$ & $\begin{array}{l}1.6 \pm 1.1(-3.8 \\
\text { to } 0.5)\end{array}$ & 0.135 \\
\hline
\end{tabular}

Values are means \pm SEM (95 \% confidence interval); $\mathrm{BP}=$ blood pressure. PT: power exercise training session; Con: control session.
Table 3

Hemodynamic responses during the power exercise training and the nonexercising control sessions.

\begin{tabular}{|c|c|c|c|}
\hline & Control & Power Training & $\begin{array}{l}\mathrm{p} \text { value PT vs. } \\
\text { Con }\end{array}$ \\
\hline \multicolumn{4}{|l|}{$\begin{array}{l}\text { Mean blood } \\
\text { pressure }\end{array}$} \\
\hline Pre & $91.0(81-101)$ & 94.7 (91-99) & 0.509 \\
\hline Leg press & $102.5(97-108)$ & $99.3(93-105)$ & 0.171 \\
\hline Bench press & $103.6(99-109)$ & $99.7(94-105)$ & 0.114 \\
\hline Knee extension & $105.2(100-111)$ & $100.3(96-105)$ & 0.014 \\
\hline Upright row & 103.4 (98-109) & $105.8(100-112)^{*}$ & 0.353 \\
\hline Knee flexion & $104.7(99-110)$ & $98.1(95-102)$ & 0.001 \\
\hline \multicolumn{4}{|l|}{$\begin{array}{l}\text { Rate-pressure } \\
\text { product }\end{array}$} \\
\hline Pre & $\begin{array}{l}8502 \text { (7887 to } \\
9118)\end{array}$ & 8504 (7914 to 9093$)$ & 0.996 \\
\hline Leg press & $\begin{array}{l}8831 \text { (8083 to } \\
9579)\end{array}$ & $\begin{array}{l}11,657(10,831 \text { to } \\
12,483)^{*}\end{array}$ & $<0.001$ \\
\hline Bench press & $\begin{array}{l}8960 \text { (8211 to } \\
9709)\end{array}$ & $\begin{array}{l}10,780 \text { (9913 to } \\
11,646)^{*}\end{array}$ & $<0.001$ \\
\hline Knee extension & $\begin{array}{l}8986 \text { ( } 8224 \text { to } \\
9748)\end{array}$ & $\begin{array}{l}12,015(11,118 \text { to } \\
12,912)^{*}\end{array}$ & $<0.001$ \\
\hline Upright row & $\begin{array}{l}8862 \text { (8184 to } \\
9541)\end{array}$ & $\begin{array}{l}12,062(11,244 \text { to } \\
12,881)^{*}\end{array}$ & $<0.001$ \\
\hline Knee flexion & $\begin{array}{l}8959 \text { (8316 to } \\
9602)\end{array}$ & $\begin{array}{l}11,212(10,388 \text { to } \\
12,036) *\end{array}$ & $<0.001$ \\
\hline
\end{tabular}

Values are means (95\% confidence interval); Mean blood pressure (mmHg); Rate-pressure product (mmHg.bpm); PT: power exercise training session; Con: control session; * $\mathrm{p}<0.05$ vs. Pre.

BP behavior and a methodology of choice to understand the time course of post-exercise hypotension in a usual living condition. ${ }^{29}$ To the best of our knowledge, this is the first trial investigating the acute effects of power training on $24 \mathrm{~h} \mathrm{BP}$. We found significant reductions in office BP at $1 \mathrm{~h}$ following $40 \mathrm{~min}$ of power exercises. However, under ambulatory conditions, only a borderline significance reduction in daytime and nighttime diastolic BP was found after the PT session. No differences between the sessions were found for $24 \mathrm{~h}$, daytime and nighttime systolic and mean BP. These findings partially confirm the results of two recent studies that found office BP reduction during $1 \mathrm{~h}$ after the power training session ${ }^{23,24}$ and add new information related to the effects of power training on $24 \mathrm{~h}$ BP in older men and women with essential hypertension.

In contrast to our working hypothesis, a single bout of power training did not reduce systolic or diastolic 24 -h ambulatory blood pressure. The absence of studies assessing the power exercise effects on $24 \mathrm{~h}$ BP limits the ability to compare our present findings with others using a similar exercise protocol. However, in studies using traditional resistance exercise protocols, $\mathrm{PEH}$ is frequently reported in the laboratory conditions (i.e., 30-90 min after exercise) but the results are controversial when $\mathrm{PEH}$ was assessed under ambulatory conditions over $24 \mathrm{~h} .{ }^{18}$ Two previous studies failed to report a decrease in ambulatory BP after a single session of traditional resistance exercise in middle-aged hypertensive men $^{30,31}$ while significant decreases in nighttime and $24 \mathrm{~h}$ systolic/diastolic BP $(\sim 4 \mathrm{mmHg})$ was found following a resistance exercise session in middle-aged overweight/obese women. ${ }^{32}$ Data in older adults that evaluated resistance exercise alone on $\mathrm{PEH}$ assessed via ambulatory BP are rare, but a combined exercise (resistance + aerobic) protocol failed to demonstrate $\mathrm{PEH}$ when $\mathrm{BP}$ was assessed under ambulatory conditions. ${ }^{28} \mathrm{~A}$ variety of factors, including an initial BP level, intensity and duration of the exercise, and exercise mode, are involved in determining both the PEH magnitude and duration in participants with essential hypertension. ${ }^{33}$ Similarly, the duration of disease and the co-existence of pharmacological treatments may also influence these outcomes. ${ }^{18,34}$ A lack of differences in ambulatory BP after the PT protocol could be due to the lower baseline BP values observed in the present study. Our sample consisted of older individuals with well-controlled hypertension who used antihypertensive medications, 
which could partially explain the smaller magnitude and duration in ambulatory BP found in our study.

The results of a meta-analysis suggested that one bout of resistance exercise can reduce ambulatory BP in hypertensive individuals, especially when large muscle groups are used during the exercise. ${ }^{18}$ However, our power training session composed of 5 exercises using large muscle groups did not reduce total $24 \mathrm{~h}$ ambulatory BP. We speculate that the total resistance/load performed during the exercise session (sets $\mathrm{x}$ repetitions/time under tension $\mathrm{x}$ load $\mathrm{x}$ exercises) may be a key component in predicting the magnitude and duration of PEH. A significant reduction in nighttime and $24 \mathrm{~h}$ systolic/diastolic BP following a resistance exercise protocol is not commonly observed but could result from a higher total overload (i.e., 19 sets of 10 repetitions at $60 \% 1 \mathrm{RM}^{32}$ than that used in the present study (i.e., 15 sets of 8-10 repetitions at 50 $\% 1 \mathrm{RM})$. Our exercise protocol provided a sufficient stress to reduce systolic/diastolic BP for $1 \mathrm{~h}$ after exercise in a controlled laboratory condition, but this reduction was not sustained under ambulatory conditions. Interestingly, the first daytime $(2 \mathrm{mmHg} ; \mathrm{p}=0.063)$ and nighttime ( $3 \mathrm{mmHg} ; \mathrm{p}=0.062$ ) diastolic $\mathrm{BP}$ had a tendency to decrease after the power training session. These results have important clinical relevance, providing a potential to reduce the cardiovascular stress during daily activities and sleeping in older individuals with hypertension.

Ambulatory BP can be affected by the time of day at which exercise is performed. Our present study is unique as exercise was performed at the same time of the day in the afternoon. Afternoon or evening exercise can increases melatonin releases during nighttime periods, ${ }^{35}$ which might lead to a decrease in nighttime BP. It is plausible to speculate that the borderline significance decrease in nighttime diastolic BP (CI $95 \%$ : -7.1 to $0.1 \mathrm{mmHg}$ ) and the non-significance decrease in nighttime systolic BP (CI $95 \%$ : -6.9 to $1.6 \mathrm{mmHg}$ ) observed in the present study could be partially explained by melatonin since it is known to modulate cardiac autonomic function ${ }^{36}$ influencing BP subsequently. These acute effects on BP have a substantial clinical implication as these acute changes are linked to chronic reductions in BP brought on by regular exercise. ${ }^{37}$ Additionally, nighttime BP is a stronger predictor of all-cause mortality and cardiovascular events than daytime blood pressure in patients with hypertension. ${ }^{38,39}$ Future studies using different combinations of exercise are necessary to confirm PEH during nighttime periods.

Office BP was lower after PT session than that obtained in Con, confirming the post-exercise hypotensive effect after PT session and the effectiveness of this alternative form of resistance exercise to acutely reduce $\mathrm{BP} .{ }^{24}$ However, exercise professionals should prescribe effective and proper resistance exercises not only to achieve maximal benefits but also to avoid exacerbated hemodynamic responses during exercise ${ }^{40}$ that increases the risk of acute adverse events especially in patients at higher cardiovascular risks. ${ }^{20}$ Slight increases in cardiac load as assessed by rate pressure products that occurred during the PT session were expected, and no adverse events occurred during the exercise session. Collectively these results suggest that resistance exercises using low intensity and repetitions not to failure such as power exercises can reduce cardiac demand during the resistance exercise session.

Poor health and disability are not the inevitable consequences of aging. ${ }^{9}$ Older individuals who adopt a healthy lifestyle is more likely to exercise, have lower health-related medical costs and a better quality of life. ${ }^{41}$ Although various modes, formats, and doses of physical activity protocols are available to reduce BP, optimal exercise prescriptions for individuals with hypertension remain controversial. ${ }^{42}$ Power exercise training involving large muscle groups used in the present study was feasible, and no adverse effects were reported during the study. The results of this trial provide evidences of acute effects of power exercises on BP management, supporting recommendations for exercise prescription in older patients with hypertension. Because power training may also promote benefits in musculoskeletal functionality, ${ }^{10,19}$ this mode of exercise may be ideal for older individuals who tend to suffer from hypertension as well as sarcopenia. Chronic studies using this exercise modality are warranted.

Some limitations should be addressed in the present study. Our present sample consisted of individuals between 60-75 years, therefore limiting the generalization of our findings to younger or older populations with hypertension. Additionally, only two familiarization sessions were performed before the strength tests. The prescribed exercise intensity may have been underestimated reducing total overload, and as consequence, influenced the PEH magnitude and duration. Strengths of the present study include the use of ambulatory BP monitoring, the goldstandard method to assess $\mathrm{PEH}$, statistical analyses using a robust method that encompasses proper adjustments that control the type I error rate using Bonferroni tests, and independent evaluators, avoiding possible analysis bias to interpret the results.

\section{Conclusion}

In summary, a single bout of PT decreases office BP but this hypotensive effect is not sustained under ambulatory conditions in older patients with essential hypertension. Future studies need to investigate the long-term effects of a power training program on $24 \mathrm{~h}$ ambulatory blood pressure and its associated mechanisms in this population.

\section{CRediT authorship contribution statement}

Renato P Schimitt: Conceptualization, Investigation, Writing original draft. Leandro O Carpes: Investigation, Formal analysis, Writing - review \& editing. Lucas B Domingues: Investigation, Formal analysis, Writing - review \& editing. Hirofumi Tanaka: Formal analysis, Writing - review \& editing. Sandra C Fuchs: Formal analysis, Writing review \& editing. Rodrigo Ferrari: Conceptualization, Investigation, Formal analysis, Funding acquisition, Writing - original draft, Writing review \& editing.

\section{Declaration of Competing Interest}

The authors report no declarations of interest.

\section{Acknowledgments}

The authors would like to thank all the participants in the study and Dr. Guilhermo Sesin for his support during the data collection. The authors also thank CAPES and CNPq Brazilian Government Associations for its support to this project.

\section{References}

1 Frontera WR, Hughes VA, Lutz KJ, Evans WJ. A cross-sectional study of muscle strength and mass in 45- to 78-yr-old men and women. J Appl Physiol. 1991;71 (1985):644-650.

2 Granacher U, Gruber M, Gollhofer A. Force production capacity and functional reflex activity in young and elderly men. Aging Clin Exp Res. 2010;22:374-382.

3 Artero EG, Lee DC, Ruiz JR, et al. A prospective study of muscular strength and allcause mortality in men with hypertension. J Am Coll Cardiol. 2011;57:1831-1837.

4 Ruiz JR, Sui X, Lobelo F, et al. Association between muscular strength and mortality in men: Prospective cohort study. BMJ. 2008;337:a439.

5 Picon RV, Fuchs FD, Moreira LB, Riegel G, Fuchs SC. Trends in prevalence of hypertension in Brazil: A systematic review with meta-analysis. PLoS One. 2012;7: e48255.

6 Picon RV, Fuchs FD, Moreira LB, Fuchs SC. Prevalence of hypertension among elderly persons in urban Brazil: A systematic review with meta-analysis. Am J Hypertens. 2013;26:541-548.

7 Chow CK, Teo KK, Rangarajan S, et al. Prevalence, awareness, treatment, and control of hypertension in rural and urban communities in high-, middle-, and low-income countries. JAMA. 2013;310:959-968.

8 Cornelissen VA, Smart NA. Exercise training for blood pressure: A systematic review and meta-analysis. J Am Heart Assoc. 2013;2:e004473.

9 Cadore EL, Pinto RS, Bottaro M, Izquierdo M. Strength and endurance training prescription in healthy and frail elderly. Aging Dis. 2014;5:183-195.

10 Fragala MS, Cadore EL, Dorgo S, et al. Resistance training for older adults: Position statement from the national strength and conditioning association. J Strength Cond Res. 2019;33:2019-2052. 
11 MacDonald HV, Johnson BT, et al. Dynamic resistance training as stand-alone antihypertensive lifestyle therapy: A meta-analysis. J Am Heart Assoc. 2016:5.

12 Kenney MJ, Seals DR. Postexercise hypotension. Key features, mechanisms, and clinical significance. Hypertension. 1993;22:653-664.

15 Liu S, Goodman J, Nolan R, Lacombe S, Thomas SG. Blood pressure responses to acute and chronic exercise are related in prehypertension. Med Sci Sports Exerc. 2012 44:1644-1652.

16 Wegmann M, Hecksteden A, Poppendieck W, et al. Postexercise hypotension as a predictor for long-term training-induced blood pressure reduction: A large-scale randomized controlled trial. Clin J Sport Med. 2018;28:509-515.

17 Pescatello LS, Kulikowich JM. The aftereffects of dynamic exercise on ambulatory blood pressure. Med Sci Sports Exerc. 2001;33:1855-1861.

18 Casonatto J, Goessler KF, Cornelissen VA, Cardoso JR, Polito MD. The blood pressure-lowering effect of a single bout of resistance exercise: A systematic review and meta-analysis of randomised controlled trials. Eur J Prev Cardiol. 2016;23: $1700-1714$.

19 Bottaro M, Machado SN, Nogueira W, Scales R, Veloso J. Effect of high versus lowvelocity resistance training on muscular fitness and functional performance in older men. Eur J Appl Physiol. 2007;99:257-264.

20 Lamotte M, Niset G, van de Borne P. The effect of different intensity modalities of resistance training on beat-to-beat blood pressure in cardiac patients. Eur $J$ Cardiovasc Prev Rehabil. 2005;12:12-17.

21 Lovell DI, Cuneo R, Gass GC. The blood pressure response of older men to maximum and sub-maximum strength testing. J Sci Med Sport. 2011;14:254-258.

22 de Sousa NM, Magosso RF, Dipp T, et al. Continuous blood pressure response at different intensities in leg press exercise. Eur J Prev Cardiol. 2014:21:1324-1331.

23 Coelho-Junior HJ, Irigoyen MC, Aguiar SDS, et al. Acute effects of power and resistance exercises on hemodynamic measurements of older women. Clin Interv Aging. 2017;12:1103-1114.

24 Machado Filho J, Machado CLF, Tanaka H, Ferrari R. Postexercise hypotension afte muscle power training session in older adults with hypertension. J Aging Phys Act. 2020:1-6.

25 Boutron I, Altman DG, Moher D, Schulz KF, Ravaud P, Group CN. CONSORT statement for randomized trials of nonpharmacologic treatments: A 2017 update and a CONSORT extension for nonpharmacologic trial abstracts. Ann Intern Med. 2017; $167: 40-47$.

26 Malachias MVB, Povoa RMSJ, Nogueira AR, Souza D, Costa IS, Magalhaes ME, 7th brazilian guideline of arterial hypertension: Chapter 3 - clinical and complementary assessment. Arq Bras Cardiol. 2016;107:14-17.
27 O'Brien E, Parati G, Stergiou G, et al. European Society of Hypertension position paper on ambulatory blood pressure monitoring. J Hypertens. 2013;31:1731-1768.

28 Ferrari R, Umpierre D, Vogel G, et al. Effects of concurrent and aerobic exercises on postexercise hypotension in elderly hypertensive men. Exp Gerontol. 2017;98:1-7.

29 Grossman E. Ambulatory blood pressure monitoring in the diagnosis and management of hypertension. Diabetes Care. 2013;36(Suppl 2):S307-311.

30 Queiroz AC, Sousa JC, Cavalli AA, et al. Post-resistance exercise hemodynamic and autonomic responses: Comparison between normotensive and hypertensive men. Scand J Med Sci Sports. 2015;25:486-494.

31 Hardy DO, Tucker LA. The effects of a single bout of strength training on ambulatory blood pressure levels in 24 mildly hypertensive men. Am J Health Promot. 1998;13: $69-72$.

32 Tibana RA, Pereira GB, Navalta JW, Bottaro M, Prestes J. Acute effects of resistance exercise on 24-h blood pressure in middle aged overweight and obese women. Int $J$ Sports Med. 2013;34:460-464.

33 Cardoso Jr CG, Gomides RS, Queiroz AC, et al. Acute and chronic effects of aerobic and resistance exercise on ambulatory blood pressure. Clinics. 2010;65:317-325.

34 Melo CM, et al. Postexercise hypotension induced by low-intensity resistance exercise in hypertensive women receiving captopril. Blood Press Monit. 2006;11: $183-189$.

35 Buxton OM, Lee CW, L'Hermite-Baleriaux M, Turek FW, Van Cauter E. Exercise elicits phase shifts and acute alterations of melatonin that vary with circadian phase. Am J Physiol Regul Integr Comp Physiol. 2003;284:R714-724.

36 Kitajima T, Kanbayashi T, Saitoh Y, et al. The effects of oral melatonin on the autonomic function in healthy subjects. Psychiatry Clin Neurosci. 2001;55:299-300.

37 Tibana RA, de Sousa NM, et al. Correlation between acute and chronic 24-hour blood pressure response to resistance training in adult women. Int J Sports Med. 2015;36: $82-89$.

38 Hansen TW, Li Y, Boggia J, Thijs L, Richart T, Staessen JA. Predictive role of the nighttime blood pressure. Hypertension. 2011;57:3-10.

39 Fagard RH, Celis H, Thijs L, et al. Daytime and nighttime blood pressure as predictors of death and cause-specific cardiovascular events in hypertension. Hypertension. 2008;51:55-61.

40 MacDougall JD, Tuxen D, Sale DG, Moroz JR, Sutton JR. Arterial blood pressure response to heavy resistance exercise. J Appl Physiol. 1985;58:785-790.

41 Yamada M, Arai H, Sonoda T, Aoyama T. Community-based exercise program is costeffective by preventing care and disability in Japanese frail older adults. J Am Med Dir Assoc. 2012;13:507-511.

42 Sakamoto S. Prescription of exercise training for hypertensives. Hypertens Res. 2020; 43:155-161. 